Cinémas

Revue d'études cinématographiques

Journal of Film Studies

\title{
Un indice pour ouvrir le jeu
}

\section{Bernard Perron}

Volume 10, numéro 1, automne 1999

\section{Cinélekta 3}

URI : https://id.erudit.org/iderudit/024805ar

DOI : https://doi.org/10.7202/024805ar

Aller au sommaire du numéro

Éditeur(s)

Cinémas

ISSN

1181-6945 (imprimé)

1705-6500 (numérique)

Découvrir la revue

Citer cet article

Perron, B. (1999). Un indice pour ouvrir le jeu. Cinémas, 10(1), 95-110.

https://doi.org/10.7202/024805ar

\section{Résumé de l'article}

L'auteur trace les grandes lignes d'une analogie très féconde entre le jeu et le cinéma narratif. Il définit d'abord succinctement les traits constitutifs de l'activité ludique instituée par le cinéma narratif. Ensuite, il en étudie les principes à travers l'analyse de Clue (Jonathan Lynn, 1985), le film adapté du célèbre jeu de société de Parker Brothers. d'utilisation que vous pouvez consulter en ligne.

https://apropos.erudit.org/fr/usagers/politique-dutilisation/ 


\section{Un indice pour ouvrir le jeu ${ }^{1}$}

\section{Bernard Perron}

\section{RÉSUMÉ}

L'auteur trace les grandes lignes d'une analogie très féconde entre le jeu er le cinéma narratif. Il définit d'abord succinctement les traits constitutifs de l'activité ludique instituée par le cinéma narratif. Ensuite, il en étudie les principes à travers l'analyse de Clue (Jonathan Lynn, 1985), le film adapté du célèbre jeu de société de Parker Brothers.

\section{ABSTRACT}

The author sketches out a very fruitful analogy between play and narrative cinema. He starts out by succinctly defining the constitutive characteristics of play activities as they are established by narrative cinema, then proceeds to a study of their principles through the analysis of Clue (Jonathan Lynn, 1985), the film adaptation of Parker Brothers' famous board game.

- Annie (au volant de l'autobus piégé) : "Quel rapport avec nous?" - Jack (debout près d'elle) : "Aucun. C'est un jeu. S'il reçoit l'argent, il gagne. S'il fait exploser l'autobus, il gagne. "

- Annie: "Et si c'est toi qui gagnes?"

- Jack: "Alors demain, nous jouerons un autre jeu."

Speed (Jan De Bont, 1994) 
S'il est à la fois tentant et périlleux d'utiliser l'élément d'un film, un fragment de dialogue par exemple, comme preuve à verser au dossier d'une hypothèse théorique relative au cinéma, il arrive que l'analogie ne soit pas sans quelque effet pratique. C'est le cas du dialogue placé en exergue, dans la mesure où il nous permet d'introduire la dimension ludique propre à l'interaction du spectateur et du cinéma narratif. C'est cette dimension ludique, c'est cette pratique du jeu, avec ses règles et ses parties en cause, que je voudrais exposer ici.

On ne compte plus les films qui mettent en scène les nombreuses formes de jeu: poker, combats de toutes sortes, jeux vidéo et de réalité virtuelle, etc. Mais ces cas n'épuisent pas la fonction du jeu au cinéma: ils n'en sont que la part visible. En effet, il se pratique au cinéma un jeu qui dépasse largement cette actualisation diégétique. Et cela, les propos tenus sur tel ou tel aspect du travail de réalisation le portent à notre attention. De manière explicite, c'est souvent au jeu qu'en appellent les critiques, voire les réalisateurs, pour caractériser la stratégie narrative d'un film. C'est le cas, notamment, du critique Éric Fourlanty et du réalisateur Robert Morin alors qu'ils devisent du film Quiconque meurt, meurt en douleur (Robert Morin, 1998):

\begin{abstract}
Le réalisateur de Requiem pour un beau sans-cour est un adepte du brouillage de pistes et de la remise en question du rôle du spectareur. Ici, plus que jamais, il faut jouer le jeu. Ou s'en aller. [Robert Morin affirme:] «Je pense que $j$ 'ai jamais encore tait de vue qui s'approche plus du réel. Je voulais faire quelque chose de déstabilisant, qui dérange plus qu'une ficrion: mais être déstabilisé pour un moment, pas savoir où on s'en va, c'est ça qu'on veut quand on va faire Le Monstre à la Ronde! (p. 26).
\end{abstract}

Owen Gleiberman offre un autre exemple dans la revue Entertainment alors qu'il compare la structure de Mission: Impossible (Brian De Palma, 1996) à " [...] un cube Rubik qui ne cesse de tournoyer" ou à un "jouet-quizz». De Palma use aussi de cette image dans USA Today: il décrit l'intrigue de son film comme un " $[. .$.$] jeu au cours duquel tout doit être décortiqué et qui$ surprend constamment les spectateurs» (Green, 1996). Dans 
l'Internet Movie Database, on compte par dizaines les synopsis où les personnages "jouent au chat et à la souris" (Broken Arrow, The Specialist, Wanted: Dead or Alive, etc.). Enfin, ce sont les sites Web de films américains qui en viennent à être conçus comme des jeux en ligne.

L'analogie entre le jeu et le cinéma narratif présente donc des effets pratiques, critiques ou théoriques féconds. Pourtant, à la différence des études littéraires, les études cinématographiques semblent n'avoir recours à l'analogie qu'en de très rares occasions. Comme le prouve un numéro de CinémAction sur le suspense (Bessalel et Gardies, 1994), les études cinématographiques se contentent le plus souvent de références fugitives à la dimension ludique des récits filmiques. Il n'y a pratiquement que les réflexions narratologiques d'André Gardies pour faire exception et proposer un certain nombre d'observations fort pertinentes. Ainsi, après avoir reconnu "Le pouvoir ludique de la focalisation" (1988), Gardies n'hésite pas à affirmer, dans L'Espace au cinéma, que ce que le film narratif propose au spectateur, «[...] c'est d'entrer dans un espace fondamentalement ludique: voir un film, c'est accepter de participer à une sorte de vaste jeu de rébus" (p. 189). Mais l'exception que représente Gardies ne diminue en rien notre surprise et notre perplexité: comment expliquer cette absence quasi complète d'une théorie du jeu dans les études cinématographiques actuelles alors qu'elle a pu présenter un enjeu pour deux des plus grands précurseurs français d'une philosophie et d'une narratologie du cinéma? Pour sa part, alors même quill tentait de circonscrire son "objet de recherche", Gilbert Cohen-Séat soulignait dès 1958 qu'en discutant du cinéma, on traite "[...] d'un langage ou d'une industrie, d'un procédé de diffusion ou d'un art, d'un moyen d'influence ou d'un jeu " (p. 53). Quant à Albert Laffay — dont on a largement popularisé l'expression «le grand imagier», mais en négligeant d'en préciser toute la portée - il ne manquait pas de souligner que la présence virtuelle cachée derrière tous les films est également un "meneur de jeu»: "Notre guide ordinaire, le “montreur d'images", reste toujours le maître du jeu» (p. 81).

Notons en toute justice que le jeu n'entretient pas des rapports exclusifs avec le cinéma narratif. S'il faut en croire 
l'anthropologue Johan Huizinga, le jeu infuse toutes les œuvres de fiction, et plus largement encore, la plupart des activirés humaines. Huizinga, qui est à l'origine d'un pan de la théorie anthropologique du jeu, soutient que l'être humain n'est pas tant un bomo sapiens - un homme qui raisonne - qu'un homo ludens, c'est-à-dire "un homme qui joue». À ses yeux, le jeu peut servir de fondement à toute description, à toute explication de l'activité humaine, qu'il s'agisse des interactions sociales, des guerres, des formes et des transformations culturelles, de la prise de risques ou de la création artistique. L'excellence, la performance, la compétition ou la concurrence, voilà autant de jeux que nous propose la société, autant de manifestations du ludique qui s'imposent à nous dans nos activités sociales. On sait combien le jeu représente, par l'imitation qu'il commande et l'imagination qu'il suscite, l'un des premiers moyens utilisés par les enfants pour raffermir leur prise sur le monde adulte. Prenons soin cependant de distinguer deux modalités de cette activité ludique : celle qui se déploie librement et qui est conçue au fur et à mesure de son déroulement (un play dirait-on en anglais); celle qui se définit par des règles qui en ordonnent le cours (un game ou une partie) ${ }^{2}$. Le cinéma narratif appartient à cette seconde catégorie.

Huizinga définit le jeu de cette façon:

Sous l'angle de la forme, on peut donc, en bref, définir le jeu comme une action libre, sentie comme "fictive" et située en dehors de la vie courante, capable néanmoins d'absorber totalement le joueur: une action dénuée de tout intérêt matériel et de toute utilité : qui s'accomplit en un temps et dans un espace expressément circonscrits, se déroule avec ordre selon des règles données, et suscite dans la vie des relations de groupes s'entourant volontiers de mystère ou accentuant par le déguisement leur étrangeté vis-à-vis du monde habituel (p. 34-35).

Entre le cinéma et cette première définition - à laquelle il faudra ajouter celle que donne Roger Caillois dans Les Jeux et les hommes - se tissent des similitudes évidentes. Malgré l'influence des médiateurs culturels (publicité, promotion, site Web, 
critique, etc.), le spectateur choisit le film qu'il verra, et il ira le voir là où il le voudra et à l'heure qu'il lui plaira. C'est cette torale liberté du spectateur qui redouble l'activité gratuite du joueur. Considérée comme un divertissement, la séance de cinéma offre au spectateur - toujours à la merci de l'ennui une possibilité d'évasion. La conscience ludique - et on y reconnaîtra le fameux clivage spectatoriel - chevauche donc deux milieux: celui de la réalité naturelle et quotidienne suspendue momentanément, et celui de la réalité artificielle qui prédomine durant le film. La première réalité peut toutefois reprendre ses droits sous le coup d'un choc extérieur, d'une infraction aux règles de la projection perpétrée par un briseur ou un négateur de jeu refusant de se joindre au groupe. Qu'un spectateur parle, qu'un autre rie alors que la scène est triste, qu'un troisième raconte la suite du film ou qu'il en dévoile les nœuds, la transgression révèle chaque fois à quel point le visionnement préoccupe tout entier le spectateur ludique. Aller au cinéma ne consiste pas simplement à "prendre place» dans un dispositif énonciatif et scénographique (Gardies, 1993) ou dans une institution (Odin, 1988), mais bien à "prendre part" au film. Le spectateur se voue au spectacle. Il pénètre la réalité artificielle et y collabore entièrement, car c'est pour lui et en lui que le jeu de la fiction a lieu. "Au fond, la distinction entre acteur et spectateur s'annule ici. L'exigence d'atteindre le jeu lui-même dans son contenu significatif est identique pour les deux " (Gadamer, p. 6). Spectateur donc, mais tout autant acteur, ou plutôt, tout autant joueur. C'est à ce point précis, là où il s'agit de rendre compte de l'activité spectatorielle, que la notion de jeu est la plus productive. Je ne crois pas qu'il existe meilleure notion pour étudier l'activité perceptive et cognirive du spectateur. Parce que, suivant Huizinga, "[...] reconnaître le jeu, c’est, qu'on le veuille ou non, reconnaître l'esprit. Car quelle que soit son essence, le jeu n'est pas matière" (p. 19). Toute activité ludique est mise en branle par l'état et la présence d'esprit des joueurs.

Pour approfondir la réflexion, je recourrai à un exemple exceptionnel, c'est-à-dire au seul film de l'histoire du cinéma issu de l'adaptation d'un jeu de société: Clue (Jonathan Lynn, 1985) '. Cas d'autant plus exceptionnel que le jeu éponyme de la 


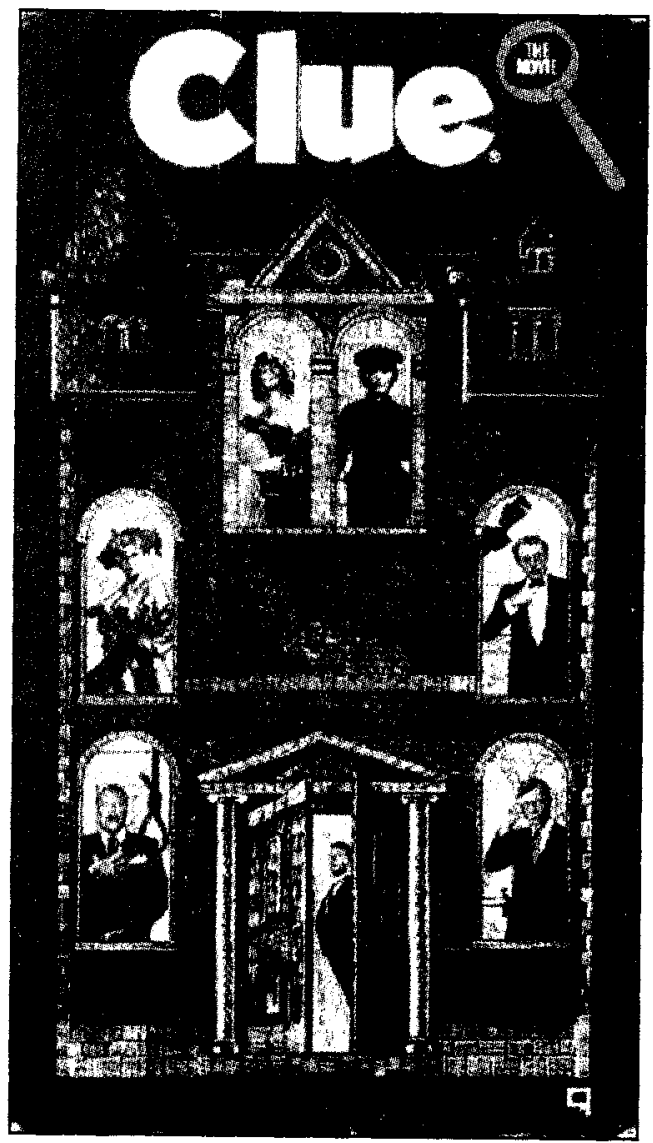

\section{Clue (Jonathan Lynn, 1985)}

compagnie Parker Brothers (enregistré en 1949) est un jeu de détective et que les histoires de détective ont constitué le modèle de référence des études littéraires portant sur la notion de jeu. En outre, le film interactif doit beaucoup à ce genre d'histoires ${ }^{4}$. Décrivons d'abord brièvement le jeu de société qui nous intéresse ici. Le système de Clue ("indice", en français) repose sur une condition préalable, le meurtre de Mr Boddy, commis dans la luxueuse demeure de ce dernier, et dont le plateau de jeu représente le diagramme. Le jeu comprend aussi six personnages identifiés comme suspects: Mr Green, Col. Mustard, Mrs Peacock, Prof. Plum, Miss Scarlett et Mrs White. En prenant place à la table de jeu, les joueurs acceptent donc une mission, 
celle d'élucider le crime. Les questions que le jeu leur impose: qui? où? comment? Les joueurs doivent démasquer le meurtrier parmi six personnages possibles, déterminer l'instrument du crime parmi six armes possibles et découvrir le lieu du crime. Le tableau de jeu consiste en un plan de la demeure de la victime et chaque joueur déplace son pion de manière à pénétrer dans l'une ou l'autre des neuf pièces, l'accès à une pièce lui conférant le droit de mener son enquête par un jeu de questions et de réponses, plutôt simples, de l'ordre de la déduction. La partie prend fin, évidemment, lorsqu'un joueur peut lever le voile sur les circonstances du meurtres.

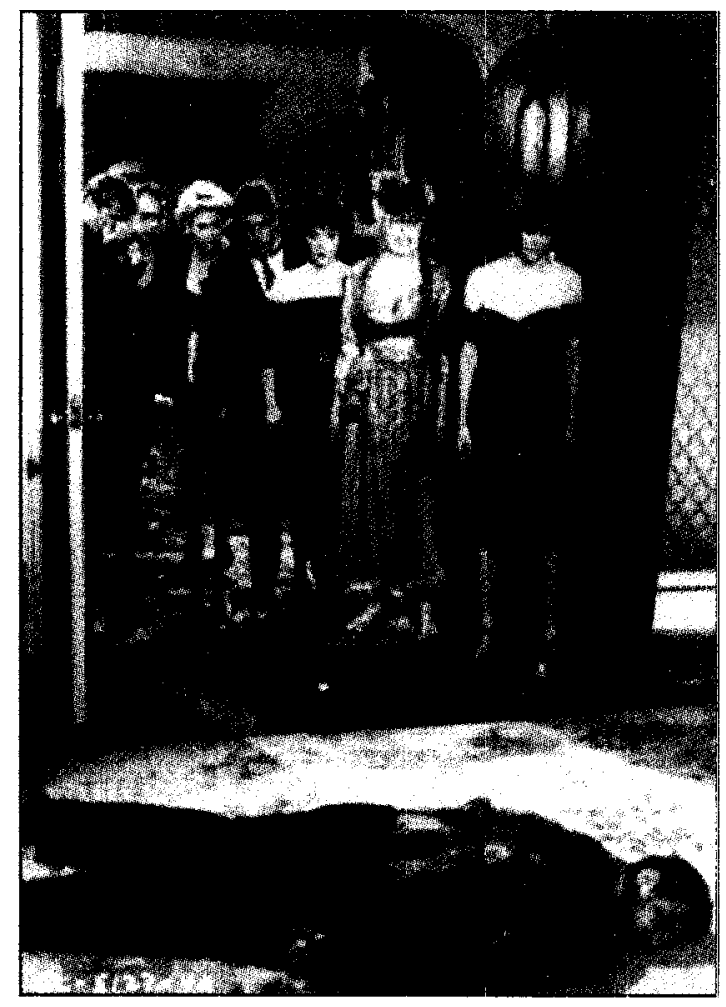

\section{Clue (Jonathan Lynn, 1985)}

Quant au film, il met en scène les sept personnages déjà connus des amateurs du jeu et en crée trois nouveaux: une bonıs, une cuisinière et, le plus intéressant pour notre propos, 
un maître d'hôtel, Wadsworth. Recevant les suspects, leur fournissant des indications, celui-ci fait figure de "meneur de jeu " intradiégétique. L'action se déroule dans un vieux manoir, terrain obligatoire du jeu et qui se constitue dans le film à travers l'univers "réservé, clos, protégé" (Caillois, p. 37) que présente le récit. Ce monde temporaire se définit aussi par les informations visuelles et sonores présentées au spectateur qui participe ainsi à sa construction. Dans le film, le spectateur n'est plus confiné aux pièces du rez-de-chaussée. Outre la route menant au manoir, il inspecte aussi, avec les personnages, la cave, le deuxième étage et le grenier. Mais bien que la salle de cinéma n'ait rien des balises et limites d'un "terrain de jeu", la durée de la séance demeure, elle, la limite temporelle imposée à l'activité ludique.

L'adaptation cinématographique de Clue va presque de soi. Dans son introduction aux règles $\mathrm{du}$ jeu, Parker Brothers note que «[...] ce jeu est différent de tout autre jeu. Tous les personnages relèvent du domaine de l'imagination - même la "victime". On se croirait au théâtre [ou au cinérma]". Si l'on voulait saisir la modalité ludique de cette adaptation à travers la classification proposée par Caillois (p. 47-74), on dirait que le rôle du simulacre (mimicry) l'emporte ici sur le hasard (alea) et le vertige (ilinx) et qu'il y entre une petite part de compétition (agôn). Outre ces quatre catégories, Caillois propose de classer les jeux selon qu'ils tendent vers deux pôles. Soit vers la paidia: "[...] un principe commun de divertissement, de turbulence, d'improvisation libre et d'épanouissement insouciant, par où se manifeste une certaine fantaisie incontrôlée" (p. 48). Soit le ludus: "[...] un besoin croissant de la plier [la paidia] à des conventions arbitraires, impératives et à dessein gênantes, de la contrarier toujours davantage en dressant devant elle des chicanes sans cesse plus embarrassantes, afin de lui rendre plus malaisé de parvenir au résultat désiré. Celui-ci demeure parfaitement inutile, quoiqu'il exige une somme constamment accrue d'efforts, de patience, d'adresse ou d'ingéniosité " (p. 48).

Les films américains à grand spectacle qui déferlent sur les écrans en période estivale tiennent de la paidia. On ne va pas voir Godzilla (Roland Emmerich, 1998), Armageddon (Michael 
Bay, 1998) ou Lethal Weapon 4 (Richard Donner, 1998) pour leur intrigue. La seule raison d'être de ces films réside dans leurs effets spéciaux (de la pyrotechnie au numérique) et, comme le dit Caillois, dans l'exubérance heureuse, l'agitation immédiate et désordonnée, la récréation primesautière et détendue qu'ils procurent. On le soupçonne, ce ne serait peut-être pas un vain rapprochement que de relire le concept de "nouveau spectateur " proposé par Roger Odin à travers celui de paidia ${ }^{6}$.

Le film Clue, en revanche, est engagé dans l'autre pôle, celui du ludus. Les histoires de détective sont des parties-jeux (des games) : le spectateur qui y joue s'attache à recueillir et à relier les morceaux d'un casse-tête, entreprise d'éclaircissement d'une énigme où jamais la clé du mystère n'est d'emblée donnée. Dès les premières mirutes du film, les personnages sont débordés par les événements et les questions fusent: pourquoi sont-ils tous invités au manoir sous des pseudonymes? qu'est-ce qui les unit? pourquoi leur fait-on du chantage? pourquoi leur distribue-t-on des armes? qui a tué $\mathrm{Mr}$ Boddy?... En conséquence, au même titre que les acteurs à l'écran, le spectateur s'engage sur les voies de la déduction pour découvrir le coupable. Il ne peut plus se fier aux «cartes visibles»: il doit plutôt tenter de percer le secret que recouvrent certainement les manœuvres des personnages. Dans le «film-jeu» (expression qu'on peut lire au sens littéral), le spectateur prend davantage conscience de son activité de lecture, des choix, des inférences, des prédictions et des rétractations qu'il effectue. Parce que de nombreux mensonges et faux (dés)aveux sont mis à nu, les affirmations des personnages et la communication narrative perdent de leur transparence et en vienrient à être considérées comme une tactique, un moyen de détourner les soupçons. À ce titre, il n'est pas inutile de rappeler l'un des "conseils intéressants" donné par Parker Brothers: "Le joueur peut même brouiller les cartes en faisant mine de soupçonner quelqu'un dont il sait trop bien qu'il ne peut être le coupable recherché puisqu'il a cette carte en main.» Mrs Peacock en fera bon usage dans l'adaptation cinématographique. Lorsque Wadsworth impute le meurtre de la cuisinière à la personne absente de la salle de billard au moment où le groupe rejoignait la bonne, Mrs Peacock — l'absente!!! - 
signale que cette personne n'aurait pu se joindre aux autres sans se faire remarquer.

Cette manière de déjouer le spectateur en lui fournissant des renseignements falsifiés ou erronés (le bluff du poker) permet de mieux définir l'action libre et "fictive" à laquelle invite le cinéma. On pourrait penser, comme le fait Gardies dans "Le pouvoir ludique de la focalisation", qu'il s'agit d'une sorte de compétition (agôn), affrontement entre le spectateur et le grand imagier. Cependant, même en anthropomorphisant l'instance racontante, on ne voit pas comment l'attitude adoptée par le spectateur à l'égard de cette dite présence virtuelle pourrait devoir quelque chose à un "[...] sentiment explicite d'émulation ou de rivalité " (Gardies, p. 80). À l'instar de la littérature, il n'y a pas de face à face effectif. Le spectateur ne peut faire le premier mouvement ni riposter. Attendu que tout jeu réclame une quelconque interaction, la construction du récit filmique doit donc comporter certains compromis et donner au spectateur des indices qui lui permettront de formuler ses soupçons. Dans le cas de Clue, c'est la voix d'une femme s'adressant à la bonne avant de l'étrangler qui sert d'indice. De la sorte, et à la différence du jeu, le film limite le spectateur à une seule question: "Qui est le coupable?" Compte tenu des limites et spécificités de l'audiovisuel, il aurait été excessif de mettre en scène tous les meurtres en se conformant au dispositif initial. Alors que la première tentative de meurtre contre Mr Boddy se produit dans une obscurité complète et fait entendre différents bruits d'armes, le film présente ensuite une mise en scène simple: le spectateur se retrouve sur quatre des six lieux du crime et l'instrument homicide lui est connu. En contrepartie, les intentions restrictives du film introduisent une incohérence narrative. Bien quaucun des personnages ne porte de gants, c'est une main gantée qui passera chaque fois à l'action: et l'image, soigneusement cadrée, limitera la part du visible. La mise en scène et la mise en intrigue du récit cinématographique, ou du simulacre (mimicry), représentent donc l'obstacle que doit contourner le spectateur. Celui-ci ne lutte pas contre un concurrent (l'adversaire), il s'escrime à franchir une chicane (l'adversité). Au premier chef, il a affaire à un ludus cinématographique. 
Puisqu'on ne franchit pas un obstacle ludique sans nécessité, l'enjeu symbolique de la partie de Clue demeure donc la résolution de l'énigme. Parce qu'elles rendent possible la parité que requiert tout jeu er qu'elles réduisent le nombre de mouvements possibles tout en instituant une liberté d'action au sein du cadre ainsi défini, less règles du jeu doivent être respectées. Parker Brothers souligne qu'il est important qu'aucun des joueurs ne voie l'une des cartes cachées, ce qui lui donnerait un atout que les autres n'ont pas. On devine que l'articulation des avantages et des désavantages perceptifs et cognitifs qu'implique la fiction cinématographique se rapproche de cette situation conventionnelle de jeu. La lecture ou le visionnement d'une histoire de détective, on le sait, repose sur certaines conventions. Notamment, rien ni personne ne doivent bouleverser le défilement linéaire du récit. Le coup d'œil furtif est interdit, le briseur ou négateur de jeu aussi. De son côté, le film doit inventer un espace propice à la réponse réglementaire. Sans remplir cette condition de manière aussi formelle que Clue, chaque film est tenu d'inscrire le ou les coupables dans une liste de suspects possibles. Le spectateur ne sait pas qui a fait le coup, mais il sait que ce ne sera pas n'importe qui. Par cette loi du genre, le film entretient des relations intertextuelles avec d'autres films policiers. L'enchâssement de la clé de l'énigme dans les horizons d'attente du spectateur favorise la reconnaissance de certains schémas narratifs et la production d'inférences. Ainsi, Jonathan Lynn emprunte entre autres à Agatha Christie les stratagèmes du Crime de l'Orient Express (1975) et du Meurtre de Roger Akroyd (1927) ${ }^{7}$. Outre cette règle de la configuration, j'en soulignerai rapidement deux autres ${ }^{8}$. D'abord, la règle de l'attention: afin de favoriser la participation du spectateur au jeu de la fiction, le film doit s'assurer que, parmi toutes les données visuelles et sonores qui se succèdent à un rythme effréné, le spectateur note celles qui importent. Les éléments sur lesquels on veut que son attention se porte doivent également posséder une certaine portée narrative. En effet, le spectateur a besoin d'une certitude, à savoir que sa compréhension du récir l'autorise à formuler des soupçons. C'est la règle de la signification. Ces règles constitutives, assimilées par le réalisateur et par le spectateur, peuvent être appliquées de façon assez souple. 
Au cinéma, l'issue du jeu est décidée d'avance. Umberto Eco l'a noté en étudiant la coopération interprétative dans les textes narratifs: "[...] en général, dans un texte l'auteur veut faire gagner, et non perdre, l'adversaire" (p. 67). On semble donc y gagner au change. Pourtant, il faut relativiser cette victoire et juger de la justesse intuitive de Laffay. Ladaptation cinématographique de Clue constitue indubitablement une pièce à conviction. L'énigme du film comporte trois clés différentes, trois finales, donc plus d'un coupable". Dans chaque cas, c'est Wadsworth qui relate les circonstances des crimes: 1) la bonne a tué Mr Boddy sur les ordres de Miss Scarlett, qui l'a par la suite assassinée avant de mettre fin aux jours du chauffeur, du policier et de la chanteuse de télégrammes; 2) Mrs Peacock est resposable du carnage; 3 ) les six suspects sont coupables d'un crime (Le Crime de l'Orient Express), à cette exception près que c'est Wadsworth, le vrai Mr Boddy, qui a tué la chanteuse ( $L e$ Meurtre de Roger Akroyd). En bout de ligne, cette triple résolution démasque bel et bien quelqu'un. Qui? Le "maître du jeu». Pourquoi? Parce que, certes, ce dernier me fait toujours gagner. Mais c'est sans que je sois en mesure d'ouvrir moi-même l'enveloppe contenant les "cartes cachées". La plus grande différence entre le film et le jeu de société, c'est que le spectateur n'a pas de bloc-notes, outil indispensable au détective, dans lequel Parker Brothers conseille de noter tous les indices recueillis.

La plus grande différence entre le film et un roman policier, c'est qu'au cours d'un visionnement normal, le spectateur doit suivre l'implacable marche des événements et des interrogations, marche ne lui permettant pas de s'arrêter pour réfléchir à un détail et pour le mémoriser s'il y a lieu. À moins de repasser le film à la loupe du magnétoscope, il lui est impossible de vérifier les circonstances des crimes. Quand Wadsworth déclare que Mrs Peacock était absente de la salle de billard et de la cuisine au moment où $\mathrm{Mr}$ Boddy et la cuisinière sont morts, le spectateur ne peut pas revenir en arrière pour scruter les deux scènes (scènes réalisées de manière à montrer sans laisser voir parfaitement les personnages qui se sont éclipsés - fin \#2). Le spectateur doit se fier à sa propre activité de construction mentale rétrospective ou aux reconstructions que le film lui impose. Ces 


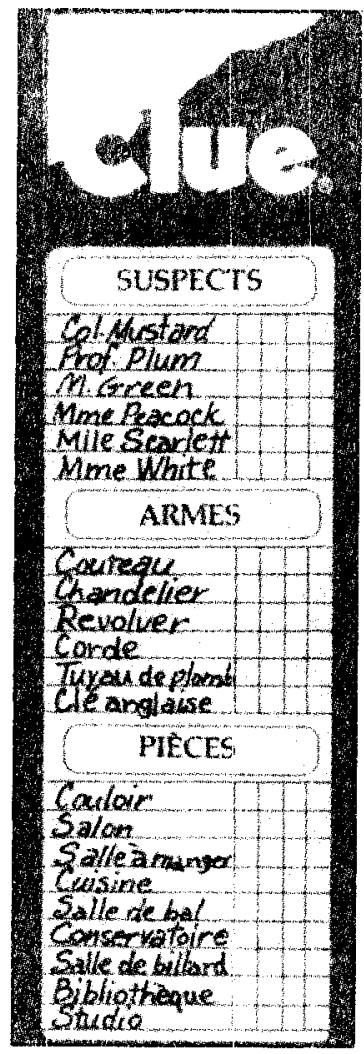

\section{Bloc-notes du jeu de Parker Brothers}

retours en arrière constituent une autre stratégie mise en œuvre par le film. Quelques minutes avant de pointer Miss Scarlett du doigt, Wadsworth propose: "Remémorons-nous cette soirée étape par étape!» Ses rappels volontaires attirent l'attention sur les faits et gestes qui viennent corroborer chacune de ses accusations, laissant chaque fois de côté ceux qui n'ont rien à voir avec elle. Par exemple, lorsqu'il accuse Miss Scarlett (fin \#1), Wadsworth ne mentionne pas que Mrs Peacock a fait une erreur fatale en révélant qu'on lui servait l'un de ses plats préférés (fin \#2). Devant de telles divergences, on ne s'étonnera donc pas que ce soient les brèches narratives que le film décide de combler et les éléments qu'il reporte à mon attention qui ont un rôle de premier plan dans la résolution de la ou des énigmes. Toutes les autres pistes demeurent brouillées. En définitive, on 
doit vouer à son "guide" (anthropomorphe ou non, préciseraisje) une confiance aveugle. Laffay avait donc raison: le "montreur d'images" reste toujours le "maître du jeu ».

Les films ne sont naturellement pas tous des "films-jeux" comme l'adaptation cinématographique du jeu de société de Parker Brothers, et ne dévoilent pas tous aussi directement la relation interactive entretenue avec le spectateur. Cependant, certains indices confirment mes "soupçons" et je vais dès lors faire une «affirmation». Ne révèle-t-on pas en narratologie qu'un récit en dit toujours moins qu'il n'en sait et ce disant, que le film trace un sentier interprétatif balayé dans une seule direction ou qu'il développe une énigme à multiples facettes. Par conséquent, le spectateur doit d'une façon ou d'une autre lutter contre l'ordre argumentatif du récit ou de la mimicry (suivant Caillois) en se servant de toute son attention, de son intelligence, de sa sagacité, de ses compétences et de ses capacités mémorielles. Bref, il doit transformer " [...] les buts de son comportement habituel en pure tâche ludique " (Gadamer, p. 33). Certains films lui rendent la vie facile en favorisant la paidia, alors que d'autres tiennent plutôt du ludus. Imperceptiblement peut-être, le jeu se glisse ainsi dans les salles de cinéma. Et celui qui y pénètre à son tour est, de toute évidence, un spectator ludens.

Université de Montréal

\section{NOTES}

1 Cet arricle fait partie d'un projet de recherche sur le jeu, la cognition et le cinéma narratif. Il a été rédigé avec le support d'une bourse postdoctorale du Conseil de recherches en sciences humaines du Canada.

2 Cette distinction entre play et game appartient à D.W. Winnicott (Jeu et réalité, Paris: Gallimard, 1975). Je la traduirai en français par "jeu-création " et "partie-jeus".

3 À ma connaissance, Clue consticue le seul film qui soit une adaptation d'un vrai jeu de sociéré. Un carton du générique de début indique clairement la source du film. Par contre, l'histoire de Jumanji (Joe Johnston, 1995) avec Robin Williams s'élabore également autour d'un jeu de société, mais ce jeu imaginaire décrit plutôt un motif diégétique.

4 En effet, les films interactifs ou jeux vidéo d'aventure contenant des séquences vidéo réelles (par opposition aux décors er aux personnages modélisés en $3 \mathrm{D}$ ) sont des histoires de détective. Je pense à Tex Murphy, Under a Killing Moon (Access Software, 1994), à The Lady Was Loaded (Philips Media, 1995) ou à The X-Files Game (Fox Interactive, 1998). 
5 Plus précisément, les réponses sont cachées dans une petite enveloppe qui contient trois cartes prises dans trois paquets différents (suspects, pièces et armes). Une fois ces trois cartes cachées, les joueurs se distribuent les autres préalablement brassées. Associés à l'un des personnages, les joueurs se déplacent sur le tableau de jeu. Une fois entré dans une pièce, un joueur peut formuler un "soupçon" en nommant un suspect, une arme et la pièce où il se trouve. Lorsqu'un joueur, par l'entremise des soupçons confirmés ou démentis, pense avoir recueilli assez d'indices et qu'il se croit capable de déduire less circonstances du crime, il porte une "accusation». S'il a raison, il est le gagnant. Sinon, il ne peut plus jouer, mais continue tout de même à intervenir afin de contredire les "soupçons" des autres.

6 Bien qu'il soit possible de faire tendre vers la paidia les films qui ne contrarient pratiquement pas la progression du spectateur, j'ai théorisé différemment et de façon plus précise l'opposition des pôles paidia-ludus dans le chapitre 7 de ma thèse de doctorat, qui propose une classification des parties-jeux filmiques (La Spectature prise au jeu. La narration, la cognition et le jeu dans le cinéma narratif, Montréal: Université de Montréal, 1997, p. 190-210).

7 En bousculant les règles du roman policier d'énigme, Le Meurtre de Roger Akroyd montre que les règles du jeu se transforment. Ce qui était considéré comme une tricherie en 1926 est maintenant perçu comme une stratégie acceptable parce qu'il fait partie des horizons d'attente du spectateur.

8 Je propose dans ma thèse un système de quatre règles constitutives reliées les unes aux autres. J'omets ici d'introduire la règle de la cohérence. Voir en ce sens le chapitre 8 au sujet de "La convention des règles du jeu" (La Spectature prise au jeu. La narration, la cognition et le jeu dans le cinéma narratif, Montréal: Université de Montréal, 1997, p. 211-233).

9 Alors que la version vidéo présente une à la suite de l'autre ces trois fins, celles-ci étaient présentées dans des salles différentes à la sortie commerciale du film et les journaux mentionnaient laquelle des salles présentait telle ou telle fin.

\section{OUVRAGES CITÉS}

Bessalel, Jean et André (iardies (direction). "Le suspense au cinéma». CinémAction, n" 71 (1994).

Caillois, Roger. Les Jeux et les hommes. Le masque et le vertige. Paris: Gallimard, 1958.

Christie, Agatha. Le Meurtre de Roger Akroyd. Paris: Librairie des Champs-Elysées, 1927.

Christie, Agatha. Le Crime de lOOrient-Express. Genève/Paris: Spadem/Cosmospress, 1975.

Cohen-Séat, Gilbert. Essai sur les principes d'une philosophie du cinéma. Paris: Presses Universitaires de France, 1958.

Eco, Umberto. Lector in fabula. Le vôle du lectetur. Paris: Éditions Grasset/Fasquelle, 1985.

Fourlanty, Éric. "Quiconque meurt, meurt en doulcur. (iarde ì vues». Voir, 26 février4 mars 1998, p. 26.

Gadamer, Hans-Georg. Vérité et méthode. Les grandes lignes d'une herméneutique philosophique. Paris: Seuil, 1976.

Gleiberman, Owen. "Choose to Accept It". Entertainment, édition électronique du 31 mai 1996.

Green, Tom. "A "Mission" Complete with Intrigue". USA Today, édition électronique c'u 21 mai 1996. 
Huizinga, Johan. Homo ludens. Essai sur la fonction sociale du jeu. Paris: Gallimard, 1951.

Gardies, André. "Le pouvoir ludique de la focalisation". Protée, vol. 16, n" 1-2 (1988), p. 139-144.

Gardies, André. L'Espace au cinéma. Paris: Méridiens Klincksieck, 1993.

Laffay, Albert. Logique du cinema. Création et spectacle. Paris: Masson, 1964.

Odin, Roger. "Du spectateur ficrionnalisant au nouveau spectateur : approche sémiopragmarique». Iris, n" 8 (1988), p. 121-139.

Perron, Bernard. La Spectature prise au jeu. La narration, la cognition et le jeu dans le cinéma narratif (thèse de doctorat). Montréal: Université de Montréal, 1997.

Wilson, Robert Rawdon. In Palamedes' Shadow. Explorations in Play, Game \& Narrative Theory. Boston: Northeastern University Press, 1990.

Winnicott, D.W. Jeu et réalité. Lespace potentiel. Paris: Gallimard, 1975. 